Im Ruhestand

\title{
Abschied von einem erfüllten Arbeitsleben
}

— Eine, die das Glück hatte, ihre persönliche Leidenschaft zum Beruf zu machen, ist Dr. h.c. Ramona Schumacher. Die gelernte Krankenschwester - seit 2004 im Dienste des Sanitätsdienstes der Bundeswehr wurde nun vom Inspekteur des Sanitätsdienstes, Generaloberstabsarzt Dr. Michael Tempel, mit einem Bestpreis ausgezeichnet und "schweren Mutes" in ihren "wohl verdienten" Ruhestand vrabschiedet.

Ramona Schumacher besetzte erstmalig die Stelle der Leitenden Krankenschwester der Bundeswehr und seit 2012 auch erstmalig die der Inspizientin Medizinalfachberufe. Sie trug maßgeblich dazu bei, dass die Gesundheitsfachberufe ein "Sprachrohr" in dem bislang approbationsdominierten Gremium der Deutschen Gesellschaft für Wehrmedizin und Wehrpharmazie e.V. gefunden haben. Damit schuf sie dieser Berufsgruppe eine starke Lobby.

In ihrer letzten Rede als Leitende Krankenschwester der Bundeswehr nahm Schumacher Bezug auf ihre "Korsettstangen", welche bis zuletzt ihr berufliches Handeln gestützt haben. Dabei standen die fachliche Aus- und Weiterbildung, klare Perspektiven in der Personalentwicklung sowie die Wei- terentwicklung der Attraktivität der zivilen und militärischen Angehörigen der Gesundheitsfachberufe stets an erster Stelle. Schumacher: "Sollten wir jetzt nicht die Weichen anders stellen, werden wir in spätestens fünf bis zehn Jahren nicht mehr das Personal zur Verfügung haben, welches wir für die qualifizierte Auftragserfüllung benötigen." Für Schumacher liegt der Schlüssel in einer zielgruppengerechten Nachwuchswerbung: „Nur wenn wir es schaffen, die Gesundheitsfachberufe als attraktiven Arbeitsplatz zu präsentieren und aktiv für den Dienst im Sanitätsdienst der Bundeswehr werben, werden wir in der Personalrekrutierung einen Fortschritt machen."

Prägend waren ihre Aufenthalte in Afghanistan und Kosovo, blickt Schumacher auf ihre zwölf Jahre im Sanitätsdienst der Bundeswehr zurück. „Diese halfen mir dabei, ein klares Bild davon zu bekommen, was unsere Kameradinnen und Kameraden Tag für Tag im Auslandseinsatz leisten und welche Anforderungen daraus im Vorfeld resultieren." Darüber hinaus waren die Zentralisierung des Kommandos Sanitätsdienst der Bundeswehr 2012 in Koblenz, die Wiedereröffnung der Krankenpflegeschule

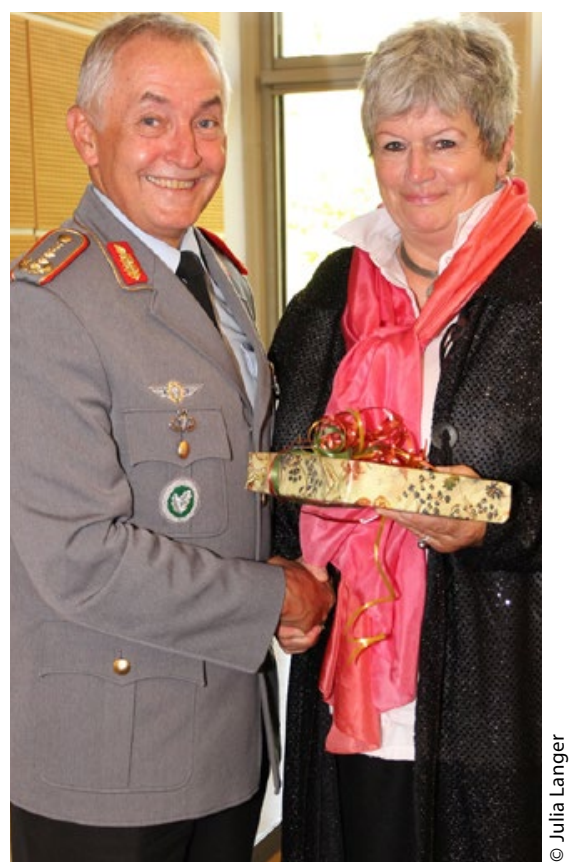

Der Inspekteur des Sanitätsdienstes, Generaloberstabsarzt Dr. Michael Tempel, verabschiedet Dr. h.c. Ramona Schumacher.

in Ulm und der Start der ersten Studiengänge für die Offiziere des Militärfachlichen Dienstes im Sanitätsdienst der Bundeswehr wichtige Stationen ihrer eindrucksvollen Karriere.

(red)

\section{Kommunen warnen \\ Kommunen warnen vor knapper Zeit}

\begin{abstract}
— Die kommunalen Spitzenverbände kritisieren Tempo und Kosten der Pflegereform. Eine geordnete Umsetzung des dritten Pflegestärkungsgesetzes sei wegen der "zeitlichen Enge" kaum möglich, formulierten Vertreter der Verbände in einer Anhörung vor dem Gesundheitsausschuss. Das Gesetz soll zum 1. Januar in Kraft treten. Die von der Regierung angeführten Mehrkosten von 184 Millionen Euro im Jahr seien zudem zu niedrig angesetzt. Grund sei ein deutlicher Mehraufwand für Sozialhilfe.
\end{abstract}

www.aerztezeitung.de

\section{Kongress in Berlin Pflege 2017}

_ Unternehmenskultur als entscheidender Wettbewerbsfaktor ist ein Thema auf dem Kongress Pflege 2017, mit dem Springer Pflege zum 22. Mal das Fortbildungsjahr für die Branche eröffnet. Der renommierte Fachkongress findet am 20. und 21. Januar 2017 im in Berlin statt.

Freuen Sie sich auf ein spannendes Fortbildungsprogramm mit zahlreichen Themen aus Recht, Management, Bildung, Praxis und Personal. Zudem steht eine zweitägige Juristische Fachveranstaltung auf dem Programm, die 2017 erstmals auch für Nicht-Juristen zugänglich sein wird. Ergänzt wird das umfangreiche Programm von einer Expertenrunde Pflegende Angehörige.

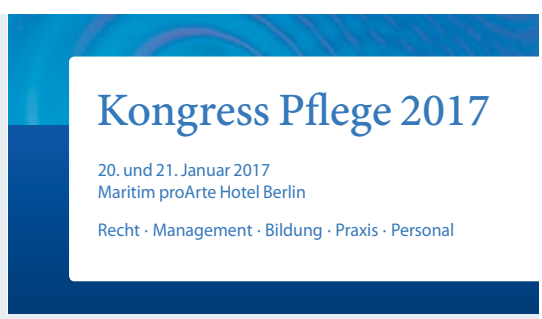

Erstmals veranstaltet Vincentz Network das Forum Altenpflege im Rahmen des Kongresses Pflege - mit aktuellen Themen rund um die professionelle Altenpflege. Glänzend wird es, wenn im ZDF-Hauptstadtstudio im Rahmen der Kongressparty der Pflegemanagement-Award verliehen wird - erstmals in zwei Kategorien. Sind Sie dabei?

www.gesundheitskongresse.de 